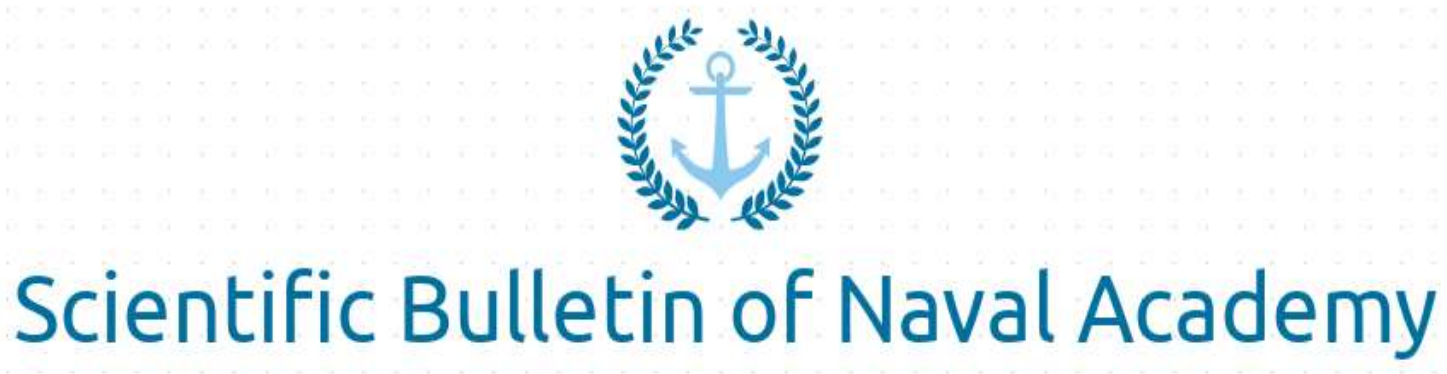

SBNA PAPER • OPEN ACCESS

Aspects of psychological training in alpine skiing at the age of 14-16

To cite this article: Cătană (Brus) Denisa-Iulia, Scientific Bulletin of Naval Academy, Vol. XXIII 2020, pg.300-310.

Available online at www.anmb.ro

ISSN: 2392-8956; ISSN-L: 1454-864X

doi: $10.21279 / 1454-864 X-20-12-108$

SBNA@ 2020. This work is licensed under the CC BY-NC-SA 4.0 License 


\title{
Aspects of psychological training in alpine skiing at the age of 14-16
}

\author{
Cătană (Brus) Denisa-Iulia ${ }^{1}$, Enoiu Răzvan Sandu ${ }^{2}$ \\ ${ }^{1}$ Dunărea de Jos University from Galați \\ E-mail: denisa.brus@,unitbv.ro \\ ${ }^{2}$ Transylvania University from Brașov \\ E-mail: razvan.enoiu@unitbv.ro
}

\begin{abstract}
The purpose of this research is to signal the importance of mental training which consists in developing those aspects of the athlete's psycho-behavioral activity that condition him an effective conduct in training both in terms of full adaptation to stress and stress, and in terms of technical and tactical perfection.

In this sense, we start from the premise that psychological monitoring in performance sports in general and in alpine skiing in general through objective investigations and evaluations, determine the achievement of superior results both in the training of athletes and in competition.
\end{abstract}

Keywords: psychology, sport psychology, psychology training alpine in skiing

\section{Introduction}

Sports performance is the result of a lasting construction starting with the initiation and continuing during the development of athletes until the autonomy of the practitioner, and the coach is the one who guides his steps in a confusing labyrinth of influential factors, becoming the architect of training and development until he manages or learns to control all the environmental and inherent variables both in the training process and in the competition. Under these conditions, "psychological intervention" is no longer just a methodology to address the various phases of athlete training, but the very key to longevity and performance in all its aspects, from interpersonal communication, the basis of the whole building, to persuasion, support, motivation and even self-motivation.

The ability to control stress is one of the major factors influencing sports performance. Repeated motivational speeches, in addition to the fact that they can become routine and therefore without effect, are often not enough, no matter how well argued, rational and understood they may seem, remaining powerless in trying to give the athlete all his means and ability to express himself. in competition. It happens quite often and is most often caused by the absence of a regular practice 
ingenuity in this regard. Stress is not a sophisticated fad, the contemplation of some absurd complexes of a caressed athlete. (Gorun, 2016)

Motivational skills, emotional control, self-assessment and self-development through the formation of the habit of having a personal diary for success, taking the sports life program, are characteristics of the psychological and behavioral picture of the athlete that can and should be as carefully trained and physical abilities, which are an essential component of high performance.

By the specificity required by the preparation for the tests in question, the trainings:

$>$ they have a rather accentuated monotony character

$>$ physically demanding, imposing a high energy consumption

$>$ during many of the trainings the athlete has limited contact with the coach

$>$ require strict compliance with a special recovery program.

In order to perform high performance, the athlete is selected from those with somatic, functional, biochemical characteristics required by the specifics of the tests. The coach organizes the trainings according to the most appropriate methodological plans, and the doctor indicates a rigorous program of nutrition, medication and recovery and / or recovery. To all this must be added the psychologist who strengthens and models those chapters in the psychological structure that will support the athlete for high performance.

"For quite some time now, psychology in general and sports psychology in particular has shown that both mental and physical abilities are trainable." (Gorun, 2016)

One of the great problems of psychological training is to meet the demands of performance sports at the international level, as it manifests itself today, depends largely on the mental qualities and mental participation of those who practice various sports.

In the conditions imposed today for performance, it is necessary, in addition to a training common to all athletes, and a strict individualization achieved in a professional sense. The planning, organization, analysis of an athlete's work takes into account his personality, the psychomotor and physical qualities of the athlete.

The most significant aspects of athletes' training, as felt by coaches, are:

$>$ the formation of psychic resistance to stress and the skills to solve unforeseen situations;

$>$ appreciation and improvement of the level of psychomotor qualities: reaction time, reaction opportunity, motor learning speed;

$>$ regulation and self-regulation of emotional tension in the pre-competitive and competitive period;

$>$ determining the personality profile of the athlete regarding the skills, temperament, character, which can influence his behavior in training and competition;

$>$ methods of psychological recovery;

$>$ analysis of mental demands in training and competitions; 
resolving internal conflicts or between athletes, between athletes and coach.

The psychological training process is organized and planned in relation to the particularities of the athlete, as performance is ensured primarily by the athlete, subjected to a certain type of effort, with biological and psychological demands, sometimes at the limit, which he bears a particular manner.

The scientific approach to training and the avoidance of unnecessary waste of physical and mental effort requires the objectification of the training process, the rationalization of the means of training, their standardization and the algorithmizing of training. That is why there is a need for work in the multidisciplinary team. (Psychological training of athletes as a factor of training, 2012, page 3)

\section{Research}

The research took place between January and April at the Edelweiss Sports Club, Brașov and consisted of applying five tests specific to a number of 20 athletes who practiced alpine skiing.

Presentation of control samples:

- Temperament test - The temperament type test has the following working procedure: two questions are given to each question. The first marked with a and the second with $b$. You think of answers which of the two best suits your usual way of being. If the answer a suits you, it means that you will add 2 to the corresponding answer, and if the answer $b$ suits you, it means that you will add 0 to the afferent answer. And if you think that your way of being is between the two answers, rather than one of them, then you will mark 1 in that box.

Processing and interpretation: the 15 questions focus on: E (emotion), A (activism) and P-S (persistence of impressions: P-primary, S-secondary). After completing the table with the answer that suits you, make the arithmetic sum of the numbers on each column as follows: add the numbers entered as answers to the questions: 1,4,7,10,13 (questions about the degree of emotion, noted with E), then add the figures entered as answers to the questions: 2,5,8,11,14 (which assesses the degree of activism, marked with A) and finally proceed similarly for the questions: 3,6,9, 12.15 (which appreciates the persistence of impressions, marked with $\mathrm{P}$ - primary and $\mathrm{S}$ - secondary). After the amount on each column has been calculated, proceed as follows: values between 0-4 mean non E non AP (nonemotive, nonactive, primary), and values between 6-10 mean EAS (emotional, active, secondary). If the value 5 is obtained on any of the columns, then the temperamental formula is written twice - once with a positive value and once with a negative value. (Self-knowledge and of others - collection of psychological tests, 1996)

- Communication style test - The working procedure is as follows: appreciate with TRUE / FALSE the following statements, as it is characteristic of you, from the 60 statements. Mark the chosen option with an "X" in the answer sheet. The result is calculated according to the following model: one point is awarded to the "TRUE" answers. The 
points are summed up on communication styles. The style in which the maximum number of points was obtained indicates the dominant attitude in communication, the relatively stable and predictable characteristics of communicative behavior. (Communication style survey, 2015 )

The test is relevant for the 4 fundamental communication styles:

$>$ Non-assertive style (passive running attitude): $1,7,15,16,17,25,26,35,36$, $37,50,51,52,59,60$.

> Aggressive style (attack attitude): 4, 6, 10, 11, 20, 21, 28, 29, 30, 39, 40, 48, 49, 55,56 .

Manipulator style (manipulation attitude): 3, 5, 9, 12, 13, 19, 22, 31, 32, 41, 42, 46, 47, 54, 57.

$>$ Assertive style (constructive attitude): 2, 8, 14, 18, 23, 24, 27, 33, 34, 38, 43, $44,45,53,58$.

- Mindset test - The way it works is this: read the statements below and appreciate to what extent you agree or disagree with each of them. It should be noted that "partially agree" does not mean simultaneously and "partially disagree": we can partially agree with a statement in the sense that it is verified only in certain situations, about the others can not say anything.( Psyhotests, 2002)

The test result is interpreted according to the following model:

$>$ Total between $\mathbf{- 6 0}$ and $\mathbf{- 3 1}$ points: you are characterized by a very strong tendency to give up, to give in to problematic situations. Pessimistic, insecure, timid, apathetic, depressed.

$>$ Between -30 and $\mathbf{0}$ points you are prone to abandonment, you trust your own strengths, you are afraid of risks, you refuse to take initiatives. Conformist, you lack energy, you prefer stability at any cost. You tend to underestimate yourself.

$>$ Between 1 and 30 points you have a winning psychology, you are enthusiastic, optimistic, dynamic, aware of your own abilities, confident in your own strengths, able to take initiatives. Well oriented and self-oriented, destined for success.

$>$ Between 31 and 60 points you have a winning psychology at any cost. You are energetic, brave, determined, passionate about risk, but sometimes arrogant, defiant, with overvaluation tendencies. The tendency to outdo yourself makes you sometimes resort to morally questionable means. Accept that there are other valuable people around you, be more cooperative, and seek to censor your dominance tendencies a little.

- Krepelin Test - This test looks at the ability to concentrate and the ability to learn and memorize, it also tests mental flexibility. It is used to investigate intellectual work capacity by evaluating the performance of performing numerical mental calculations.

The working procedure is as follows: you have to

\begin{tabular}{|l|l|}
\hline FB - Very good & $81-100$ \\
\hline B - Good & $61-80$ \\
\hline M - Medium & $41-60$ \\
\hline S - Satisfying & $21-40$ \\
\hline NS - Nesatisfying & $1-20$ \\
\hline
\end{tabular}
find and circle the pairs of numbers that together give "10". For example the pair of numbers "1" and "9" or "9" and "1". You circle these 
pairs directly on the Grid. We are not looking for pairs of numbers on the vertical, we are only looking for pairs of numbers on the horizontal. The maximum working time is 10 minutes. The result is calculated according to the following model: for each pair found a point is added, and for each pair missed / omitted / not found a point is deducted.

- Prague Test - This is a test that is applied collectively and was developed by the Prague Psychotechnical Institute by adapting the individual form, unsuitable for use when it is necessary to perform rapid measurements on large groups, especially in the environment. industrial. The test is recognized as the "Prague" Distributive Attention Test. Of course, it can also be administered individually. The test also aims at topographic memory, the spirit of observation, resistance to mental fatigue generated by repetitive activities. The working procedure is as follows: there are 4 columns of numbers on the "Answer sheet" tab. On the "Grid" tab, find some squares, with pairs of numbers, two kinds of numbers: large bold numbers and small thin numbers.

The first number in the first column of the "Answer Sheet" is 59. Look for the number 59 in the "Grid" tab in each square among the large numbers in bold. Below the number 59 you will find the number 70, written in thin letters. You will go to the first column of the "Answer sheet", next to number 59, number 70. Do the same with the other numbers in the first column and in the following columns. When you finish one column, move on to the next. The maximum working time is 15 minutes. The result is calculated according to the following model: each correct answer, each number-pair association of letters, is worth 1 point. Of the total pairs of letters identified, erroneous / wrong pairs will not be considered. The results obtained after applying the Prague test, offer us the opportunity to appreciate the level of distributive attention of the athlete, as well as his resistance to mental fatigue.

\begin{tabular}{|l|l|}
\hline FB - Very good & $81-97$ \\
\hline B - Good & $61-80$ \\
\hline M - Medium & $41-60$ \\
\hline S - Satisfying & $21-40$ \\
\hline NS - Nesatisfying & $1-20$ \\
\hline
\end{tabular}

\section{Presentation and interpretation of research data}

Following the temperament test applied to all evaluated athletes, regardless of age, it turned out that most of them have a passionate temperament, which means that they have a focus on the result. They are also ambitious people who achieve what they set out to do, have a unique purpose, are strong, balanced, serious and have good leadership qualities, but they can also be domineering or stubborn. The passionate style is closely followed by the phlegmatic and amorphous style. The phlegmatic type is focused on principles, and the amorphous type on pleasure. The phlegmatic type is calm, friendly, persevering, has a critical sense, is objective, weighted and has a critical sense, but at the same time can be cynical, calculated, comfortable, slow and typical. Instead, the amorphous type is the man of the present, attentive, tenacious, but he is careless, careless, regardless of past and future. At the other end, the fewest athletes tested have a nervous temperament, focused on entertainment and an apathetic temperament, focused on silence. 
Depending on the type of temperament that each athlete has, the coach must use the appropriate mode for each type of temperament. For example, for the passionate guy, the coach should give him responsibilities, not be ironic with him and last but not least give him reasonable explanations. It can be seen from the chart below that most athletes between the ages of 14 and 16 have a passionate type of temperament, which means that they focus on the result and should be encouraged to get the best results possible.

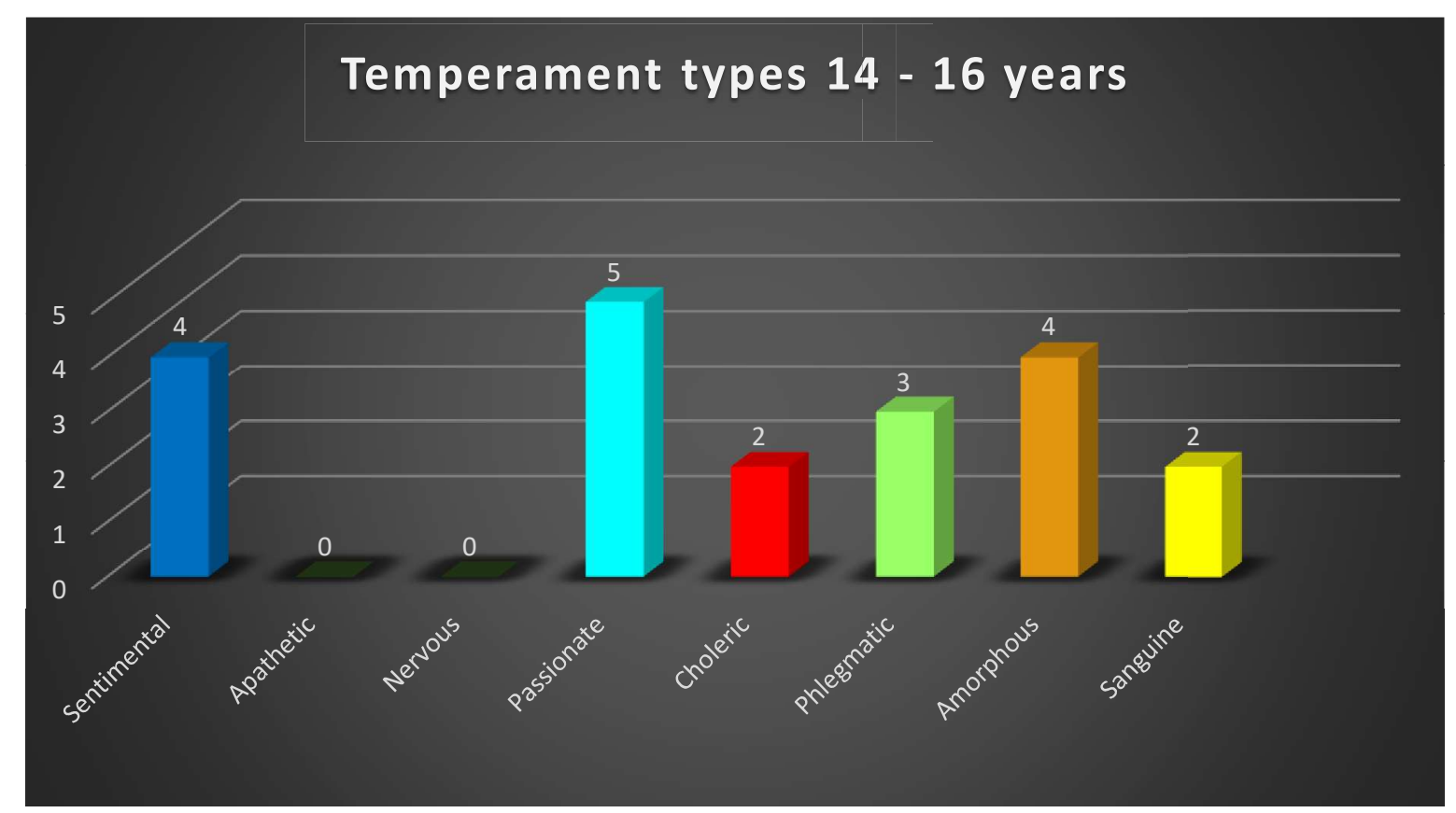

Fig.1. Results regarding the determination of the temperament of the sample 14 - 16 years $(\mathbf{n}=\mathbf{2 0})$

After studying the types of temperament of athletes and also discussing with coaches and athletes how to approach each so that they evolve as well and efficiently and also have better and better results, $80 \%$ of them had unexpected results.

Following the test to establish the style of communication through which we can figure out how to process information and transform this information into behavioral facts, practical, social, evaluative judgments, etc. we conclude the following. Most subjects have a predominantly assertive communication style, which means that they have the ability to self-affirm honest, direct and clear expression of their opinions and rights without aggression and without harming others. Also, the person knows how to listen and is willing to understand, knows how to be himself (without simulations and "role-playing games") and to rely on himself. This is the best attitude because it allows you to achieve your goals without provoking the resentment of others and even gaining their sympathy. 
In the case of athletes aged between 14 and 16, it is mainly the assertive style, which means that it is the best attitude and communication style.

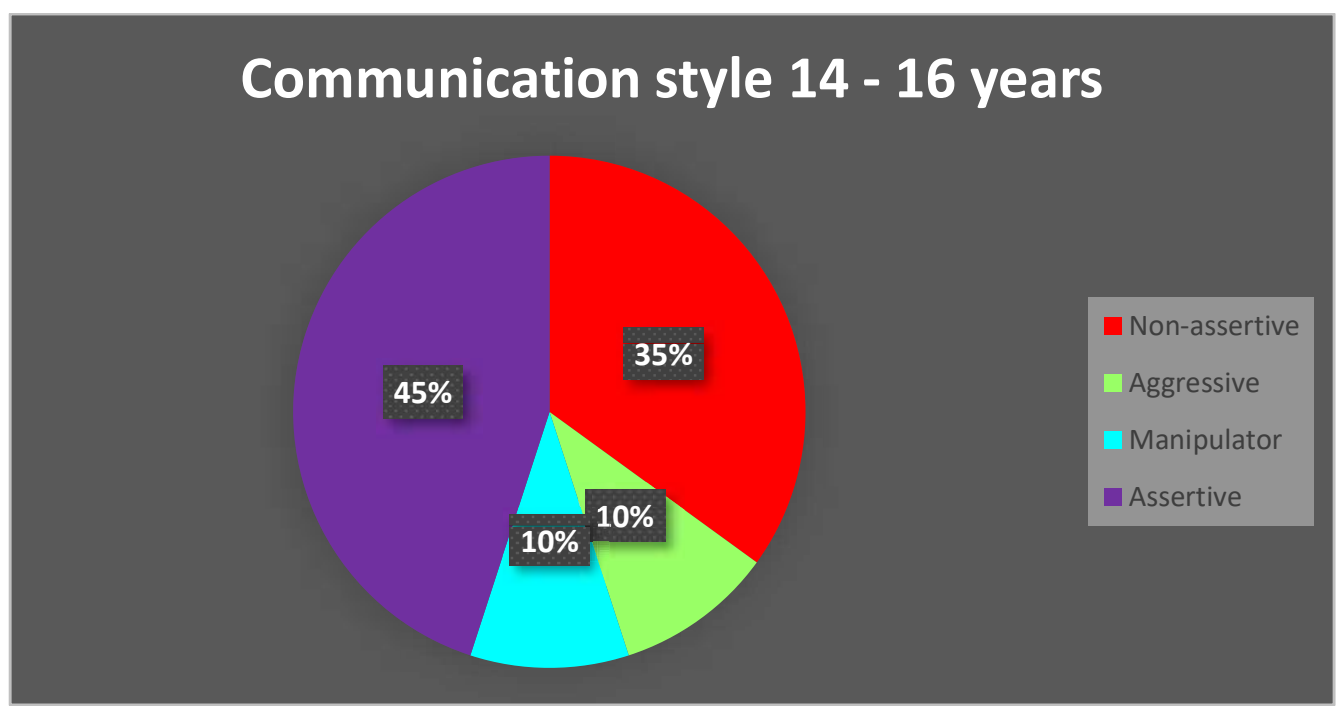

Fig.2. Results on determining the communication style of the sample 14 -16 years $(\mathbf{n}=\mathbf{2 0})$

Through the winning psychology test we were able to find out more about the mentality with which athletes approach problematic situations and about their attitudes towards performance. This test showed that most of the test subjects have a winning psychology, which means that they are optimistic, dynamic, aware of their own abilities, self-confident and able to take initiatives, the test is intended to assess success.

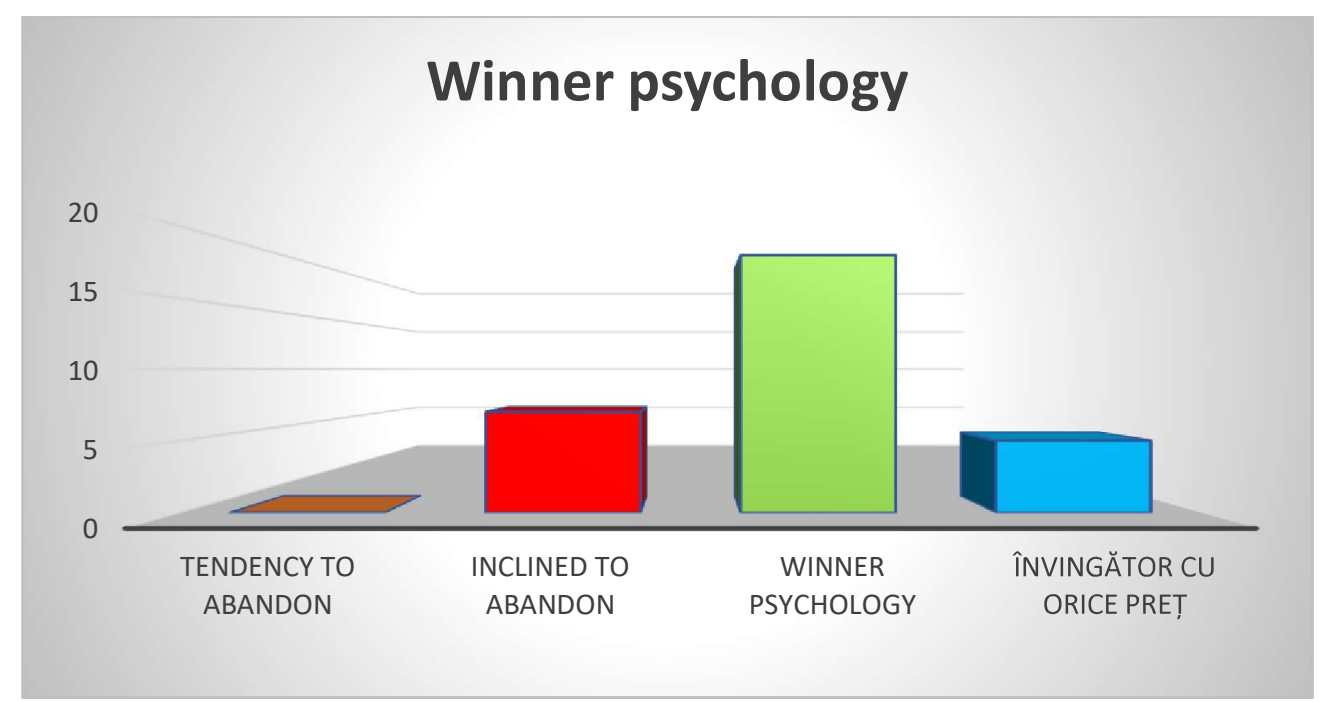

Fig.3. The results regarding the determination of the winning psychology of the studied sample $(n=20)$ 
In order to have a broader view of the test, after finding out its results, we subjected the athletes to a game. The game consists of breaking a pencil with one finger. What I wanted to see through this game was that those who after the test resulted in a winning mentality should not give up until I break the pencil, and those who are prone to abandonment to give up after a few tries. Before trying to break the pencil, each athlete was asked what the chances are of breaking the pencil, most answering that they have a $50 \%$ chance of breaking the pencil, and some of them answered that the chances of breaking the pencil are $1 \%$. Those who gave the lowest percentage are in the category of those prone to abandonment. I found that some of those with a winning mentality gave up without breaking the pencil. The results of the game were as follows:

$>$ in the case of the athletes in the winning category at any cost, all the athletes broke the respective pencil. In the case of some of them, the pencil did not break at first, but they did not let go until they broke it;

$>$ in the case of athletes in the category of winning psychology, not all subjects who participated in this game managed to break the pencil. Some of them gave up after several unsuccessful attempts. Hence the fact that they trust their own strengths, but are afraid of failures;

$>$ in the case of athletes in the category inclined to abandon the vast majority of them as expected after several unsuccessful attempts they gave up. But there were also 3 athletes who, even if they didn't succeed for the first time, tried until they succeeded, they didn't give up the fight;

The result of the game was that the test was largely correct, with a few exceptions, which is gratifying, because in this way the coach can make those in the category prone to abandonment to be winners.

The Kraepelin test aims at the ability to concentrate attention and the ability to learn and memorize, it also tests mental flexibility.

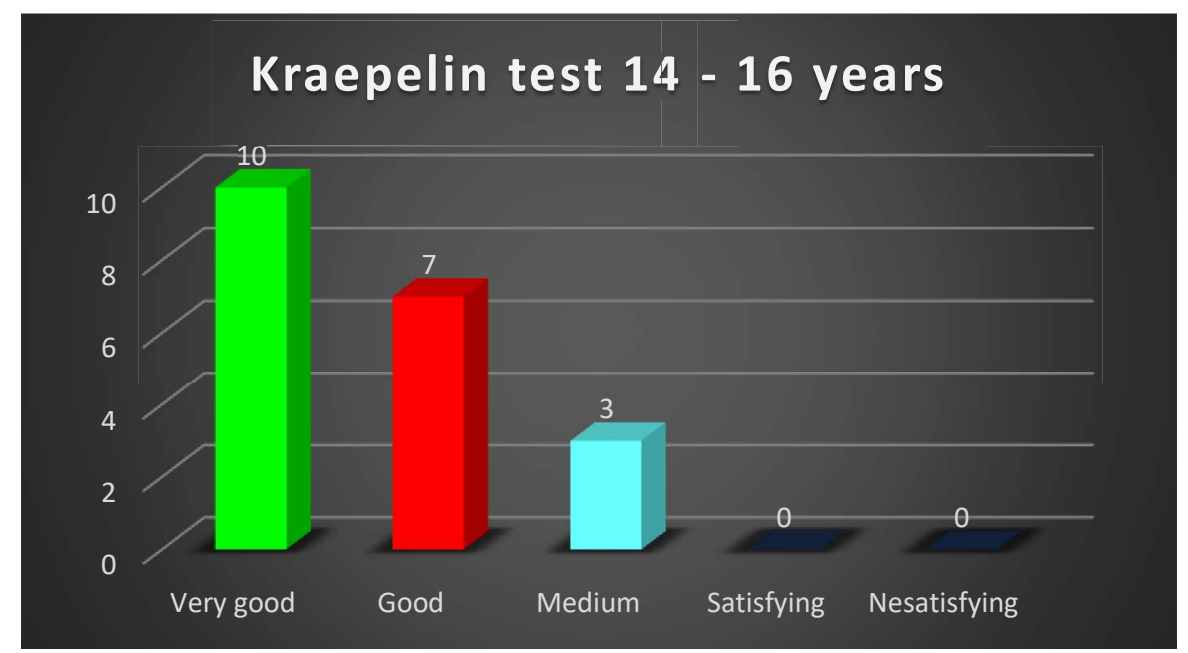

Fig.4. Results regarding the determination of the concentration capacity of the sample 1416 years $(n=20)$ 
In the case of athletes aged between 14 and 16, they mainly have a very good learning and memorization capacity and an equally good mental flexibility. In the case of young athletes, the coach together with the sports psychologist must work so that all athletes reach a very good learning and memory capacity, in this way they will get better and better results.

The last test the athletes underwent was the Prague test which is recognized as the "Prague" Distributive Attention Test. The test also aims at topographic memory, the spirit of observation, resistance to mental fatigue generated by repetitive activities.

In the case of athletes aged between 14 and 16, they mainly have a resistance to mental fatigue generated by repetitive activities and a very good topographic memory, which shows that they are well prepared mentally, which helps them in competitions. . On the other hand, athletes who have obtained a medium or satisfactory grade must work together with the coach and the sports psychologist, through games, so that their results become better.

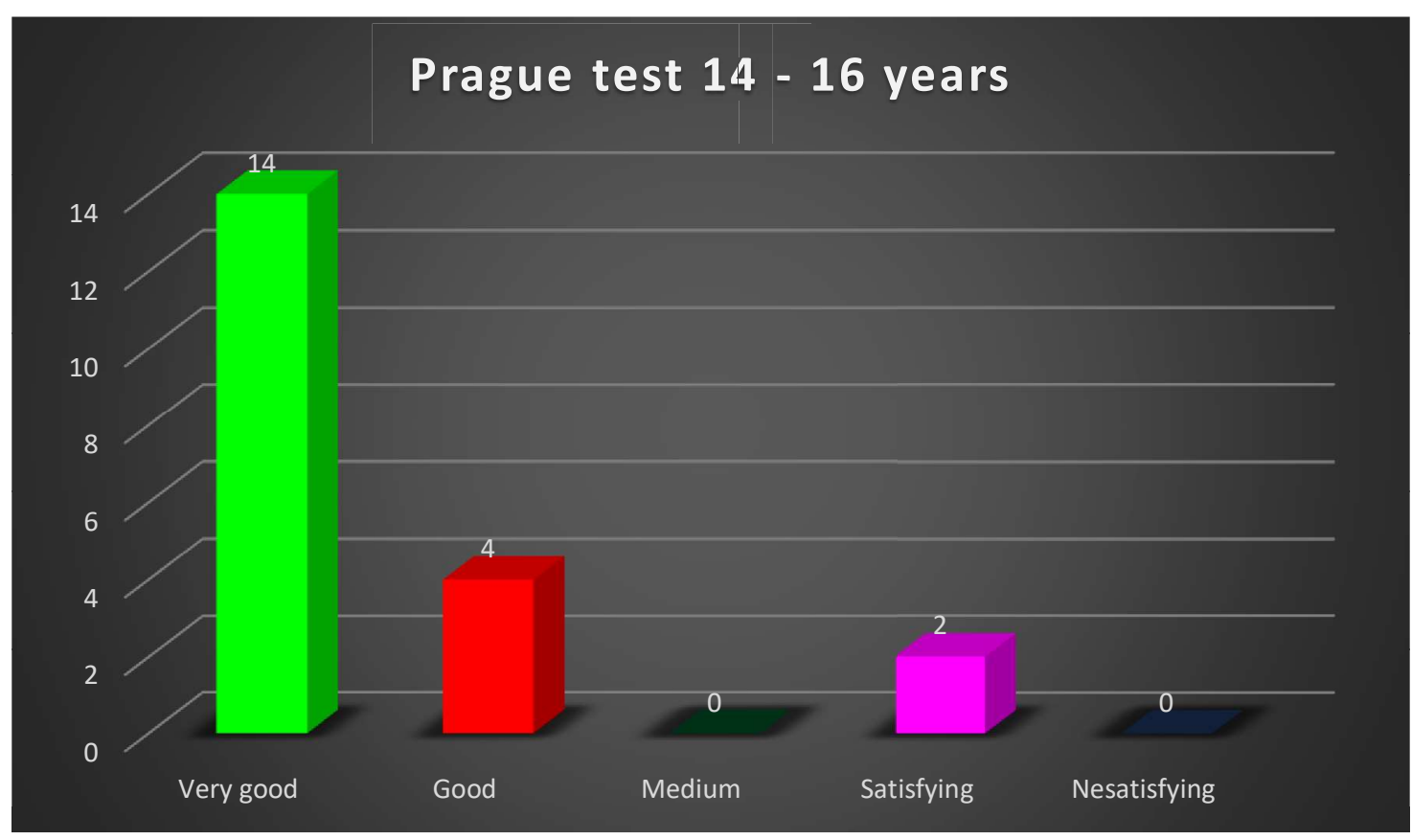

Fig.5. Results on the determination of the distributive attention of the sample 14-16 years

$(\mathbf{n}=\mathbf{2 0})$ 


\section{Conclusions}

Following the tests applied to the subjects by age categories, it is found that the mobilization of psychic energy helps to combat stress, and this can generate a greater resistance to mental fatigue, due to repetitive activities and very good topographic memory.

$>$ Rest is needed to acquire a topographic memory. In this way, athletes improve their ability to concentrate. Focusing on a specific element leads to improved sports performance.

$>$ The increase of the psychic energy contributes to an increase of the attention so the expected results will be clearly superior within the competitions.

$>$ Through continuous psychological training and a positive thinking approach, most athletes can get good results in competitions.

$>$ From my point of view, I consider that the better the nervous system, the better the effort of both the coach and the athlete.

In order to be able to establish a coherent, realistic strategy with concrete effects on athletes, it is necessary to know how to approach it, as well as their characteristics according to age.

From the presented, it can be concluded that the major role in the performance of athletes is played by their age. This is the main reason why the tests performed in this paper were aimed at two age categories, in order to establish working methods to increase performance in athletes.

Psychic barriers are doubly conditioned: social and individual. From a social point of view, they are determined by the influence of the group's opinion and individually, the barriers are constituted by the multitude of limiting factors that lead, through repetition, to the formation of a subjective attitude of distrust, fear. Psychic barriers in borderline states are not only affectivemotivational or motor, but also intellectual. Lack of imagination and creativity acts as an obstacle that the individual can only overcome very hard, through stimulating-activating elements: encouraging suggestion, motivation, cognitive restructuring, adjustments of the level of affectivity, restructuring in technique and tactics.

Reached the highest level, the difference is very small between the levels of training achieved by athletes and the most important factor that decides the winner is the ability to cope with psychological pressure. These factors include motivation, effort, concentration, confidence, but an important factor is the ability to withstand the stress of competition.

Many successful athletes emphasize the psychological side of the sport. Swimmer Mark Spitz, who won seven gold medals at the Olympics, is quoted as saying, "At this level of physical qualification, the difference between winning and losing is $99 \%$ psychological." Jack Nicklaus, the famous golfer, stated that "golf is 90\% mental" (Williams, 1986). Of course, the percentage values should not be taken literally, especially when an athlete talks about an increase of $150 \%$. The idea is that world-class athletes see psychological factors as very important. 
Psychological influences come into play during the treatment of problems (such as tension or sudden inability to perform at the usual level of the player) and also in relation to peak performance.

All these conclusions lead to the finding that the application of age tests shows us that in the test group aged 14-16 years the resistance to mental fatigue generated by repetitive activities is very good, they also have a very good topographic memory, communication is assertive.

The practical-methodical recommendations resulting from the tests are the following:

$>$ teachers must design their psychological training planning according to the needs of the subject;

$>$ psychological training must take into account the particularities of age, sex, stage of training;

$>$ psychological training must be closely related to the qualities and defects of the opponent;

$>$ psychological training must be related to the qualities and defects of one's sportsmanship;

$>$ in complicated situations the teacher-coach must collaborate with the specialists in the field;

$>$ Positive thinking is the most important factor in training the athlete in extreme situations.

$>$ Be careful! Be optimistic! Be Present!

\section{References}

[1] Bonchiș, E., Self-knowledge and of others - collection of psychological tests, Universitatea din Oradea, Oradea, 1996

[2] Gorun, I., (2016, 14 may), Psychological intervention in performance sports, Attitude blog about sport and society. Available at: https://sportlogic.wordpress.com/tag/interventiapsihologica-in-sportul-de-performanta/.

[3]Mihaela Andreea, (2015, 7 iunie), Communication style survey (analysis of communication style), Dream.Believe. Do. Repeat (blog). Disponibil la:

https://mihaelaandreearadu.wordpress.com/2015/07/07/chestionarul-s-c-analiza-stilului-decomunicare/

[4] Pitariu, H., Chelcea A., Psyhotests, $3^{\text {rd }}$ Edition, Ed. Societatea Știință \& Tehnică S.A., Bucharest, 2002

[5] Psychological training of athletes as a factor of training, Universitatea de Vest Vasile Goldiș Arad, Faculty of Education Sciences, Psychology, Physical Education and Sports, Specialization: Sports and Motric Performance, Arad, 2012 